Revue d'histoire de l'Amérique française

ZWB REVUE D.HISTOIRE DE L'AMÉRIQUE FRANÇAISE

\title{
Le premier livre de comptes du Séminaire de Québec
}

\section{Honorius Provost}

Volume 16, numéro 1, juin 1962

URI : https://id.erudit.org/iderudit/302168ar

DOI : https://doi.org/10.7202/302168ar

Aller au sommaire du numéro

Éditeur(s)

Institut d'histoire de l'Amérique française

ISSN

0035-2357 (imprimé)

1492-1383 (numérique)

Découvrir la revue

Citer cet article

Provost, H. (1962). Le premier livre de comptes du Séminaire de Québec. Revue d'histoire de l'Amérique française, 16(1), 37-42. https://doi.org/10.7202/302168ar d'utilisation que vous pouvez consulter en ligne.

https://apropos.erudit.org/fr/usagers/politique-dutilisation/ 


\section{LE PREMIER LIVRE DE COMPTES DU SÉMINAIRE DE QUÉBEC}

L'histoire est comme un diamant de qualité. Sous un abord terne et peu agréable, à l'état brut, elle recèle d'innombrables facettes et celles-ci découvrent leurs feux graduellement, sous la main du joaillier qui les taille. Il n'y a pas de document du passé humain qui, dûment replacé dans son contexte, ne puisse enrichir un tant soit peu quelque domaine de la culture. A l'anthropologue, à l'historien, le soin et le devoir de mettre en valeur le plus possible de cette richesse native, pour le bénéfice de leur génération et de celles qui viendront après, jusqu'à l'extinction de l'humanité sur notre planète. Dans l'autre monde, on n'aura plus besoin de l'histoire...

Voici que nous avons en main, pour l'analyser, le premier livre de comptes du Séminaire de Québec: à première vue, une masse sans consistance de données hétéroclites, souvent énigmatiques, parfois, du reste, indéchiffrables. Nous lui soupçonnons bien quelque potentialité. Mais à l'instar du statuaire de Lafontaine, nous nous demandons d'abord: "Sera-t-il dieu, table ou cuvette ? 》 Pour le moment, il nous pèse au bout du bras, ce vieux registre couvert de peau blanche, épais de trois bons pouces et mesurant treize pouces par neuf, sur les deux autres dimensions. Le dernier tiers du registre n'a pas servi. Tout ce beau papier perdu, alors qu'il était pourtant si précieux! Il y a exactement 600 pages d'utilisées d'ailleurs souvent incomplètement.

Ce livre de comptes s'étend sur la période de 1674 à 1683 . En fait, si c'est là le premier livre de comptes qui nous reste, il porte, bien en évidence sur le dos, l'inscription: « $2^{\mathrm{e}}$ livre de Raison », selon l'ancienne terminologie. Et des entrées, par-ci par-là, nous réfèrent à un livre précédent qui a dû avoir au moins trois cents pages. Hélas ! il est disparu, sans dire comment ni pourquoi, comme le chat de la mère Michel. Qui est-ce qui nous le rendra? 
De qui sont, à présent, les entrées de notre livre de comptes, s'échelonnant sur une période de dix ans, au jour le jour, selon le caprice des recettes et des déboursés ? La réponse, un enfant la donnerait: elles sont du procureur du Séminaire. Bien ! Mais il reste à savoir qui était le procureur, ou, plus exactement, quels ont été les prêtres agissant comme procureurs durant cette période?

Le premier procureur du Séminaire, depuis sa fondation, ce fut l'abbé Jean Dudouyt, excellent homme d'affaires et teneur de livres, soit dit en passant. Il partit cependant, en novembre 1676, délégué par Mgr de Laval auprès du Roi et de Colbert. En attendant son retour, car il ne se proposait pas de rester en France, ce qui advint effectivement, on lui donna un substitut dans la personne de l'abbé Pierre de Francheville, nouveau prêtre de quelques semaines, le premier Canadien de naissance à remplir une charge au Séminaire. Au bout de onze mois, lui succéda pour un an l'abbé Louis-Pierre Thury, qui, malgré ses quelque trente-trois ans d'âge, n'était encore qu'à la veille de son ordination. Mais ces deux prêtres, nous semble-t-il, n'ont dû être que des teneurs de livres au nom d'un autre; et de qui ? Peutêtre de Mgr de Laval lui-même, dont l'abbé de Francheville était sûrement le secrétaire.

A tout événement, on ne retrouve un procureur en titre, dans la personne de l'abbé François Fillon, qu'en novembre ou décembre 1678, au moment même où $\mathrm{Mgr}$ de Laval part à son tour pour l'Europe. Il doit y avoir là plus qu'une coïncidence. M. Fillon disparaît tristement dans une noyade, au bout de six mois, et l'on voit revenir M. Thury, cette fois procureur en titre, jusqu'à 1683, alors que, partant pour le ministère, il est remplacé par l'abbé Jean-François Buisson de Saint-Côme. Comme on le voit, cela fait plusieurs règnes en un si court espace de temps; et, pour démêler l'écheveau, à défaut d'autres points de repères, il a fallu procéder à des recoupements et à des comparaisons d'écritures. La paléographie est un art indispensable en histoire.

Selon la terminologie de l'ancienne comptabilité, le livre de comptes que nous étudions se classait sous l'étiquette de Grand 
Livre, sans doute par oposition à Journal, Caisse, etc. Et ce "grand livre », le seul à survivre, ce n'était pas tant les comptes du Séminaire directement, que les comptes de divers particuliers avec le Séminaire. La rubrique uniforme est la suivante: un tel doit au Séminaire: c'est le débit; il lui est dû par le Séminaire: c'est le crédit. A ce système-là, il n'y a pas moyen, à peu près, de savoir combien le Séminaire recevait ou dépensait par année, ce qui eût été fort intéressant. Mais ce qu'on perd en concentration dans les données, on le regagne en information; et l'on est stupéfait de constater combien le Séminaire de Québec, à peine encore âgé de dix ans, pouvait avoir déjà de relations et de rayonnement. Il est vrai que cette période, 1674 à 1683, est celle des grandes constructions du Séminaire et marque une exploitation plus intense de ses domaines agricoles. Cela justifie au moins pour une part la multiplicité des opérations financières. Il y a exactement, dans notre livre, cent quarante comptes différents.

Classons maintenant en catégories les clients du Séminaire, pour ainsi parler. Dans bien des cas, d'ailleurs, l'expression est juste; car le Séminaire jouait le rôle de marchand ou de fournisseur, à cette époque où l'argent était rare et où les échanges se faisaient en nature bien plus qu'en espèces. L'histoire économique, la sociologie, la civilisation même d'autrefois s'inscrivaient dans les vieux livres de comptes, bien mieux que dans les livres tout court. Il nous est impossible, malheureusement, d'entrer dans tous les détails. L'énumération est suffisamment longue, complétée de quelques commentaires.

1. Institutions: Collège des Jésuites, Hôtel-Dieu de Québec (hôpital et communauté séparément), Ursulines de Québec, Séminaire de Montréal, Confrérie de la Sainte-Famille. Le Frère Joseph Boursier, procureur du Collège des Jésuites, était en transactions continuelles avec le procureur du Séminaire: remèdes, livres de classe, ustensiles, fournitures de toutes sortes; et la bonne bière des Jésuites n'est pas l'item le moins important. Dans le compte du Séminaire de Montréal, il est surtout intéressant de retracer les allées et venues de ces Messieurs ; à Québec, 
ils étaient bien reçus au Séminaire, mais ils payaient honnêtement leur pension...

2. Églises: Château-Richer, Sainte-Anne de Beaupré, l'AngeGardien, Sainte-Famille, I.-O., Saint-Pierre, I.-O., Saint-Paul (devenue St-Laurent), I.O., Saint-Jean-Baptiste, I.-O., Lauzon, Champlain, Trois-Rivières, Sorel, Boucherville. La raison des affaires du Séminaire avec tant de paroisses tient du fait que leurs curés ou desservants étaient des prêtres dépendant du Séminaire pour leur subsistance. Mais, dans plusieurs cas aussi, le Séminaire a financé la construction même des églises, celles de Sainte-Anne et de Sainte-Famille presque en entier. L'église de Québec ne figure pas dans la liste, bien qu'elle fût unie juridiquement au Séminaire. Mais elle avait ses comptes particuliers, conservés encore aux Archives de la paroisse.

3. Fonctionnaires: le comte de Frontenac, l'intendant Duchesneau (très peu de choses pour ces deux-là), Charles Bazire et Charles Aubert de Lachenaye, successivement agents pour la transmission des octrois royaux au Séminaire et à la Fabrique de Québec: enfin «Messieurs les Intéressés de ce Pays 》 (La Compagnie Oudiette), qui, depuis 1681, donnèrent au Séminaire une gratification de 420 livres par année, prise à même les droits d'entrée chargés par eux sur les vins, eaux-de-vie et tabacs.

4. Notables: Claude Auber, Pierre Boucher de Boucherville, Claude Bermen de La Martinière, Martin de Saint-Aignan, Philippe Gaultier de Comporté, François Hazeur, Jean Juchereau de La Ferté, Olivier Morel de La Durantaye, Denis-Joseph Ruette d'Auteuil. Parmi eux, certains étaient de bourgeoisie plutôt que de noblesse authentique. D'ailleurs, on pouvait ici commercer sans déroger d'aucune façon.

5. Affaires de terrains: Mme Guillaume Couillard, Louis Jolliet, François Fortin, Jacques Gamache dit Lamarre, Massé Gravel, Barthélémy Verreau, Pierre Vivier dit La Musette. Terrains achetés, terrains vendus; hypothèques, rentes et autres affaires de cette catégorie.

6. Fournisseurs spéciaux: Pierre Parent de Beauport, pour la pierre de taille: on employa la pierre de rang, dans les grandes 
constructions du Séminaire, mais seulement pour les encadrements de portes et de fenêtres; Pierre Maufet, blanchisseur du Séminaire, «sur le pied de dix livres par personne du grand séminaire et de huit livres, du petit séminaire; on fournit pour les deux 24 briques de savon par année ».

7. Fermiers et meuniers: Louis Jobidon, François Naud dit La Verge et Julien Allard dit La Verge, fermiers du Petit-Pré; Pierre de La Faye dit Mouture et Jacques Goulet, meuniers à Château-Richer; Pierre Gagnon, père et fils, Jean Gagnon et Romain Trépagny, fermiers au Cap Tourmente; Claude Bouchard, Noël Simard dit Lombrette et Pierre Tremblay, fermiers à la Baie Saint-Paul; Charles Pouliot, meunier à l'Ile-d'Orléans, puis au Sault-à-la-Puce; Abel Turcot, fermier à l'Ile-d'Orléans ; Guillaume Labelle et Olivier Charbonneau, fermiers à l'Ile-Jésus. Le Séminaire s'était réservé dans ses seigneuries plusieurs métairies qu'il louait à des fermiers, soit à moitié des revenus, soit à prix fixe. On passait généralement un bail notarié, pour les affaires de cette importance; mais il s'en suivait toute une comptabilité pour diverses transactions de débail.

8. Donnés: Apé, Jean; Baillon, Antoine; Beaudoin, (?) ; Dumesnil, Guillaume (?) ; Girard dit Champagne, Etienne; Lafontaine, Geoffroy; Lefranc Toussaint; Louzon, Pierre; Michel, Olivier (?) ; Patenôtre, (?) ; Quittard, Jean; Roger, Charles. L'institution des donnés a débuté en 1675 , pour être consacrée officiellement en 1678. Ces hommes et femmes s'engageaient par contrat à travailler toute leur vie pour le Séminaire, sans rénumération, mais avec la garantie de leur entretien, en santé ou en maladie. On en a gardé pendant environ un siècle. La formule était héritée des Jésuites, qui l'avaient introduite au profit de leurs missions huronnes.

9. Engagés et domestiques: On connaît la distinction entre ces deux catégories de gens. Mais, dans bien des cas concrets, il est impossible de faire cette distinction avec certitude. Nous ne donnerons donc qu'une seule liste, par ordre alphabétique, pour le bénéfice des chercheurs, généalogistes et autres, et nous signalerons le métier, lorsqu'on l'a mentionné. Il serait même possible d'indiquer le salaire convenu, le temps de l'engagement 
et, dans plusieurs cas, la date de débarquement à Québec; mais il faut nous limiter à l'essentiel: Alary, René, charpentier; Baillif, Claude, tailleur de pierre; Bissonet, Pierre, menuisier; Blain, François; Bodin, Pierre (?) ; Boislard, Jean, tireur de pierre; Bonniot, Pierre; Bouteiller, Jean (?) ; Brûlé, Julien, cordonnier; Chaillot, Jacques; Charlot dit La Mare, René; Chazart, François, charpentier; Clopestre, Guillaume, cordonnier ; Cochard, Nicolas ; Constantineau, Louis (?) ; Coutron, André, maçon; de Cardenas, P.-A., peintre; de Chaume, André; de Hauvie, David; Dubosq, Jean, jardinier; Duplais, Louis, maçon ; Duplais, Sylvain, maçon; Fauchois, Michel, apprenti-sculpteur; Ferland (Freland), François; Fourré, Benedict, tailleur de pierre; Frérot, Guillaume; Gaboury, Jean; Gagnon, François; Genner, Samuel, sculpteur; Genouzeau, Jean-Baptiste; Genouzeau, Michel ; Guignart, Pierre; Guyet, Jean, cuisinier; Hamelin dit La Chaise, Nicolas, jardinier; Henne dit Portugais, Martin; Heuvelé, Marc, tailleur de pierre; Jourdain, Guillaume, maçon; La Caude, Pierre (?) ; Langlois, Jacques, tailleur; La Ronde, (?), charretier; Latte, Pierre; Laurent, François; Lavergne, François ; LeBlond, Michel, dit le Picard, menuisier; Lefebvre, Charles; LeGrand, Pierre, cordonnier; LeGros, Jean ; LeMaire, Louis ; LeMaistre, Denis, tailleur; LeRoux, François; L'Espinay, Joseph, jardinier; L'Evesque, Robert, charpentier; L'Oiselière dit Lafleur, Jean, cordonnier; Massieu (Mathieu ?), Thomas; Morier, Jean; Perré dit Larivière, Michel; Perrier dit Baujelois, Pierre; Pommier, Michel; Prévost, Jean; Prud'homme, François, menuisier ; Quereken, Léonard, menuisier; Rivaut, Jacques; Rivière, Pierre, menuisier; Roberge, Denis ; Sénéchal, François, charpentier ; Suavet, Joseph; Til, Alexandre, tailleur de pierre; Vallois, Jacques; Verdier, Louis, couvreur d'ardoises; Villeneuve, Pierre.

HoNORIUS PROVOST, ptre Université Laval, Québec. 